\title{
A Experiência Religiosa/Espiritual de Lésbicas, Gays e Bissexuais: Uma Revisão Integrativa de Literatura*
}

\author{
Mattos Dourado de Mesquita Estrázulas ${ }^{1, * *}$ (D), \& Normanda Araujo de Morais ${ }^{1}$ \\ ${ }^{1}$ Universidade de Fortaleza (UNIFOR), Fortaleza, CE, Brasil
}

\begin{abstract}
RESUMO - Buscou-se caracterizar a produção científica acerca da experiência da religiosidade/espiritualidade (R/E) em lésbicas, gays e bissexuais (LGBs), via revisão integrativa de literatura nacional e internacional, no período de 2005 a 2015. A busca foi feita nas bases de dados SciELO, PePSIC, LILACS, IndexPsi, PsycINFO, PUBMED e E-journals, sendo identificados e analisados 58 artigos após a consideração dos critérios de inclusão e exclusão. Foram encontrados exclusivamente, estudos internacionais, predominando artigos norte-americanos, empíricos, transversais e qualitativos. As principais categorias de análise qualitativa versam sobre: integração $\mathrm{R} / \mathrm{E}$ e homossexualidade; $\mathrm{R} / \mathrm{E}$ como fator de risco e/ou proteção para LGBs; homonegatividade internalizada; e prática clínica. Ressalta-se a contribuição do estudo para a literatura nacional, bem como a relevância da R/E na vivência de LGBs.
\end{abstract}

PALAVRAS-CHAVE: religiosidade, espiritualidade, LGB, homossexualidade

\section{The Religious / Spiritual Experience of Lesbians, Gays and Bisexuals: An Integrative Review of the Literature}

\begin{abstract}
The objective is to characterize the scientific production regarding the religious/spiritual experience among lesbians, gays, and bisexuals (LGBs) from an integrative literature review, both national and international, from 2005 to 2015. The authors reviewed data from SciELO, PePSIC, LILACS, IndexPsi, PsycINFO, PUBMED, and E-journals. Therefore, they identified and analyzed 58 articles that took into account the inclusion and exclusion criteria. The main categories of qualitative analysis relate to integration between religiousness/spirituality and homosexuality, and religiousness /spirituality as a risk and/or protection factor for GLBs, internalized homonegativity, and therapeutic context. The studies highlight the contribution to the national literature scene, as well as the importance of religion/spirituality in the life of LGBs.
\end{abstract}

KEYWORDS: religiosity, spirituality, LGB, homosexuality

Os números apresentados pelo Instituto Brasileiro de Geografia e Estatística (IBGE, 2010), mostram que mais de $90 \%$ da população brasileira se declara filiada a alguma religião, o que sugere que ser religioso, ou se dizer religioso é considerado importante para os brasileiros. Quando se fala em religiosidade e espiritualidade (R/E), muitos autores (Bowland, Foster, \& Volsler, 2013; Bruscagin, 2004; Heerman, Wiggins, \& Rutter, 2007) apontam diferenças entre os dois termos. A religiosidade é definida como um sistema de crenças organizado, com valores morais, crença na existência de Deuses - ou Seres Superiores-, e que convoca as pessoas a formarem uma comunidade e partilharem da mesma fé (Bruscagin, 2004).
A espiritualidade, por sua vez, é considerada como um fenômeno existencial, que pode ser vivenciada dentro ou fora de uma comunidade religiosa formal (Bowland et al., 2013; Bruscagin, 2004; Heerman et al., 2007; Jefrries \& Sandfort, 2014; Louceiro, 2007; Pinto, 2009; Tan, 2005; Walsh, 2005).

As pessoas que vivem realidades religiosas e/ou espirituais tendem a experimentar e compreender o mundo, sua identidade e interação com os outros de acordo com os pressupostos dessas experiências (Bruscagin, 2004). Alguns dos valores tradicionais religiosos oferecem uma estrutura de referência moral, legislando, inclusive, sobre a homossexualidade (Cohen \& Savin-Williams, 2014).

\footnotetext{
* Apoio: FUNCAP

** E-mail: floramdm@gmail.com

n Submetido: 06/04/2017; Revisado: 13/11/2017; Aceito: 16/02/2018.
} 
Ainda hoje, para algumas igrejas, a homossexualidade é vista não só como pecado, mas também como doença que precisa e tem cura (Bastos, 2014; Natividade, 2006; Rodrigues, 2009), à despeito do posicionamento da ciência sobre a temática. Esta, por meio da American Psychology Association (APA), Organização Mundial de Saúde (OMS), Conselho Federal de Psicologia (CFP) e Manual Diagnóstico e Estatístico de Transtornos Mentais (DSM), por exemplo, definem a homossexualidade como uma das possibilidades de vivência afetivo-sexual humanas e não como patologia (Mesquita \& Perucchi, 2016). Supõe-se, assim, a existência de tensões entre lésbicas, gays e bissexuais (LGBs) envolvidos na seara religiosa. Essa tensão estaria presente não só na comunidade religiosa, mas também nos próprios indivíduos LGBs, que podem considerar sua orientação homossexual e a dimensão religiosa/espiritual como algo contraditório (Wilkerson et al., 2012).

No cenário nacional, a temática da religiosidade/ espiritualidade de LGBs é tratada a partir, principalmente, da Sociologia, que tende a dar ênfase a dois aspectos: a visão das instituições religiosas acerca da homossexualidade e a realidade das igrejas inclusivas. Acerca do primeiro, a literatura descreve quatro posturas provenientes das instituições: a rejeição à homossexualidade, considerando que esta é pecado e antinatural; a acolhida, desde que os homossexuais reconheçam e se empenhem em modificar seu comportamento, por meio da vivência do celibato ou da mudança de orientação sexual; a postura de aceitação da conduta homossexual, mas considerando-a inferior ao modo de vida heterossexual; e, por fim, as que defendem que ambas as formas de sexualidade têm o mesmo nível de dignidade (Busin, 2011; Carvalho, 2014; Machado,
Barros, \& Piccolo, 2010; Vilaça \& Oliveira, 2015). Acerca da realidade das igrejas inclusivas no Brasil, trata-se de um fenômeno recente, datado do final dos anos 1990, quando alguns grupos articulavam discussões acerca de religiosidade e homossexualidade em suas igrejas de origem. A partir daí, no início dos anos 2000, surgiram diversas denominações religiosas inclusivas no Brasil, que buscam lidar com a diversidade sexual e, para além de trazerem novas interpretações das escrituras, elaboram estratégias políticas em luta dos direitos humanos para lésbicas, gays, bissexuais e transexuais (Ferreira \& Silva, 2015; Jesus, 2010; Natividade, 2010; Nunes, 2013; Souza, 2013). Os estudos nesse campo se desenvolvem por meio da discussão de questões de gênero e flexibilidade para a diversidade sexual na organização eclesiástica dessas instituições. Portanto, ainda é incipiente na Psicologia nacional, estudos que tenham a R/E de LGBs como foco, fato que pode estar relacionado à dificuldade em considerar a experiência religiosa/espiritual dos indivíduos como algo que deve ser objeto de estudo da ciência psicológica (Domínguez et al., 2015; Oswald, 2002).

Diante desse contexto, e considerando que a religiosidade e a espiritualidade seguem tendo grande importância na vida de indivíduos homossexuais (Tan, 2005), realizou-se uma revisão integrativa da literatura, com o objetivo de delinear a produção científica, nacional e internacional, sobre a experiência religiosa/espiritual de LGBs, considerando o período de 2005-2015. Nesta revisão, o foco foram aqueles estudos que privilegiavam a percepção dos próprios LGBs sobre a sua religiosidade/espiritualidade (R/E) e não a fala de outras pessoas/profissionais (e.g. religiosos, familiares, médicos e psicólogos) sobre essa dimensão.

\section{MÉTODO}

Tratou-se de uma revisão integrativa da literatura, a qual buscou descrever a produção científica sobre a experiência religiosa/espiritual de lésbicas, gays e bissexuais, por meio de uma metodologia sistemática de busca, seleção e análise de artigos indexados em bases de dados eletrônicas. A revisão permitiu delinear o estado da arte sobre o assunto em foco e apresentar as possibilidades de novas investigações (Scorsolini-Comin, 2014).

A obtenção dos dados: A busca bibliográfica foi realizada nas bases de dados: SciELO, PePSIC, LILACS, Index Psi, PsycINFO, PUBMED e E-journals. Foram utilizados os seguintes descritores de busca com operadores booleanos: "(homossexual OR lésbica OR gay OR bissexual OR lgbt) AND (religiosidade OR espiritualidade OR fé religiosa OR fé OR psicologia espiritual)". Os mesmos descritores foram utilizados na língua inglesa "(homosexual OR lesbian OR gay OR bisexual OR lgbt) AND (religiosity OR spirituality OR religious faith OR faith OR spiritual psychology)".
A pesquisa nas bases de dados aconteceu nos meses de janeiro e fevereiro de 2016. Dessa forma, estabeleceu-se o período entre 2005 e 2015 como referência, com o intuito de mapear as publicações mais recentes, dada as transformações legais, jurídicas, sociais e acadêmicas em relação ao tema da homossexualidade nos últimos dez anos, como por exemplo, a aprovação da união estável, casamento, e da presença de ambos sobrenomes na certidão de crianças quando no processo de adoção por casais constituídos de pessoas do mesmo sexo (Oliveira, 2015).

$\mathrm{Na}$ revisão foram utilizados os seguintes critérios de inclusão: artigos indexados; teóricos e empíricos, em inglês ou português e disponíveis em sua versão completa. Posteriormente foram analisados os resumos (abstracts), e foram selecionados apenas os que tratavam do tema da homossexualidade relacionada à experiência religiosa/ espiritual. Após uma primeira seleção, realizada pelo exame dos resumos de acordo com os critérios de inclusão e exclusão, os artigos foram recuperados por completo e, 
nesse momento, foram lidos na íntegra. A extração dos dados foi armazenada em planilhas do Excel.

\section{Análise de Dados}

Para a síntese e interpretação dos resultados, realizaramse a partir de dois tipos de análises. A primeira, de natureza quantitativa, buscou caracterizar a produção científica, considerando as questões: ano de publicação; nacionalidade das instituições dos primeiros autores; idioma; tipo de estudo; delineamento; método; amostra/participantes; instrumentos; e categorias de análise.

$\mathrm{Na}$ segunda análise, de natureza qualitativa, organizouse e fez-se uma síntese dos conteúdos temáticos, tal como proposto por Bardin (1977/1995) e somente a posteriori mapearam-se quatro grandes categorias de análise: 1) estratégias de integração religiosidade/espiritualidade e homossexualidade; 2) religiosidade/espiritualidade como fator de risco e proteção para LGBs; 3) homonegatividade internalizada; e 4) prática clínica.

\section{RESULTADOS E DISCUSSÃO}

Identificou-se mediante a busca inicial nas bases de dados um total de 1758 estudos. A maior parte desses estudos tratava de temas relacionados ou à espiritualidade/ religiosidade ou à homossexualidade, sem, entretanto, estarem conectados entre si. Após a leitura dos resumos aplicou-se os critérios de inclusão e exclusão, restando 710 artigos, que traziam questões acerca de religião e espiritualidade relacionados com homossexualidade. Destes, 639 artigos foram excluídos por não abordar o tema da experiência da religiosidade/espiritualidade em homossexuais a partir de suas perspectivas. Muitos desses artigos traziam o ponto de vista de jovens universitários acerca de gays religiosos, ou a incidência de homofobia em locais onde a religião é presente de forma tradicional. Os 71 artigos foram lidos na íntegra e desses, 13 estudos foram excluídos após a leitura por não incluírem a perspectiva dos sujeitos LGBs sobre a sua experiência religiosa. Por fim, foram incluídos 58 estudos que compuseram o corpo de análise deste trabalho, sendo: PUBMED $(n=43)$; E-Journal $(n=8)$ PsycINFO $(n=5)$; e LILACS $(n=2)$.

\section{Caracterização Quantitativa das Produções Científicas}

Enfatiza-se que todos os estudos encontrados e analisados foram internacionais e redigidos no idioma inglês ( $\mathrm{n}=58$ ). Quanto ao período das publicações, verificase que, desde 2005, tem havido um crescente no número destas; de forma que o maior número de publicações foi verificado em $2015(n=19)$ e $2014(n=10)$. Quanto às instituições de origem dos autores dos artigos, a maioria é proveniente dos Estados Unidos $(\mathrm{n}=39)$, seguida pelo Reino Unido $(\mathrm{n}=6)$. Não há nenhum artigo de autores oriundos de instituições da América Latina ou do Brasil. Acerca das questões metodológicas foram recuperados 54 artigos empíricos e 4 teóricos. Predominaram as abordagens qualitativas $(n=34)$, seguida pelas quantitativas $(n=19)$. Apenas cinco artigos utilizaram a abordagem multimétodos. Sobre os instrumentos de coleta de dados, nos estudos qualitativos, foram utilizados, predominantemente, entrevistas estruturadas e semi- estruturadas. Para a análise dos dados, no geral, realizou-se a análise de conteúdo por temas. Nos estudos empíricos quantitativos, sobressaiu-se o uso de escalas e questionários padronizados. $\mathrm{O}$ tamanho das amostras/participantes dos estudos variou de 1 a 24626 pessoas. Pesquisas com gays $(n=14)$, e LGBs $(n=14)$ predominaram nesta revisão. Apesar de não ter sido utilizado o descritor transgênero na busca nas bases de dados, sete estudos incluíram esta população ao lado de lésbicas e/ou gays e/ou bissexuais (e.g. Bradshaw et al., 2015; Dehlin et al., 2015; Gibbs \& Goldbach, 2015; Mavhandu-Mudzusi \& Sandy, 2015).

\section{Caracterização Qualitativa das Produções Científicas}

A análise qualitativa dos estudos teve como critério a classificação dos artigos que abordavam o conjunto de temas relacionados às experiências religiosas/ espirituais de lésbicas, gays e bissexuais, destacando-se os seus principais resultados. Quatro temas emergiram da análise qualitativa. São eles: (1) Estratégias de integração religiosidade/espiritualidade e homossexualidade $(\mathrm{n}=28)$; (2) Religiosidade/espiritualidade como fator de risco e/ ou proteção para LGBs ( $\mathrm{n}=22)$; (3) Homonegatividade internalizada ( $n=4)$, e (4) Prática Clínica $(n=4)$.

Estratégias de integração religiosidade/espiritualidade (R/E) e homossexualidade. Via de regra, lésbicas, gays e bissexuais atravessam um período de autoaceitação e validação de sentimentos quanto à sua orientação sexual, quando esta é descoberta. Essa passagem tende a ser mais complicada quando esses indivíduos cresceram em contextos religiosos que professam que a homossexualidade é imoral e indesejada. Ainda que essa passagem possa levar a um distanciamento da vida religiosa, verificou-se que a religiosidade segue tendo papel importante na vida de LGBs (Tan, 2005).

Sendo assim, a literatura revisada nesse estudo tende a visibilizar dois movimentos nessa população. O primeiro diz respeito à elaboração de diferentes formas para facilitar 
o convívio harmonioso entre orientação sexual e vida religiosa/espiritual. $\mathrm{O}$ segundo diz respeito à eleição de formas de vida que confirmam que a convivência pacífica entre religiosidade/espiritualidade e homossexualidade é inviável. A essas diferentes estratégias utilizadas pela população LGB para lidar com as dimensões da R/E e orientação sexual, nomeia-se "estratégias de integração". Nessa revisão foram identificadas oito estratégias, descritas na sequência:

A primeira estratégia é saída do armário seletiva. Ela ocorre quando os indivíduos homossexuais acreditam que a igreja na qual participam não seja ciente de sua orientação sexual e, assim, preferem manter a orientação sexual em segredo nesse contexto para fugir de uma possível discriminação (García, Gray-Stanley, \& RamirezValles, 2008; Jeffries et al., 2014; Liboro \& Walsh, 2015). Além disso, pretendem evitar consequências indesejadas socialmente, como perda de emprego e consequências relacionadas à família, não desejando serem fonte de desonra, desgraça, vergonha e constrangimento (Figueroa \& Tasker, 2013; Liboro \& Walsh, 2015). Importante pontuar que tais sentimentos resultam da experiência do preconceito e discriminação de que são vítimas, de forma que é importante não naturaliza-las.

A segunda estratégia de integração refere-se à utilização da rede de apoio social, visto que a aceitação da família e/ou amigos aliviariam o fardo de uma religiosidade apreensiva e abrandaria a culpabilização (Figueroa \& Tasker, 2013; Shilo, Yossef, \& Savaya, 2015; Stamatoulakis \& Nearchou, 2015). Conhecer outros pares na mesma situação, em alguns casos através de grupos de apoio online, foi considerado como uma rede de ajuda àqueles que se sentiam únicos em seu conflito de identidade (Lalich \& Mc Laren, 2010; Siraj, 2012).

A participação em igrejas inclusivas ou com pessoas de dentro da congregação que não percebiam a homossexualidade como problemática representa a terceira estratégia de integração identificada nos estudos (Bowland et al., 2013; García et al., 2008; Itzhaky \& Kissil, 2015; Jeffries et al., 2014; Wood \& Conley, 2014). Essa estratégia se mostrou uma saída eficiente para muitos sujeitos, uma vez que as igrejas inclusivas são aquelas nas quais as sexualidades não heterossexuais (LGBs) são compatibilizadas com as religiosidades cristãs, ou seja, não julgadas como moralmente inferiores ou pecado. Essas igrejas são majoritariamente evangélicas (Jesus, 2010).

A quarta estratégia denomina-se mudança na percepção/ foco. Nela estão inseridas quatro formas significativas de mudança de percepção/foco do sujeito LGB, seja sobre a teologia, religião, homossexualidade e congregações religiosas, a saber:

a) Ressignificar a teologia e apresentar uma escuta seletiva. forma como muitos indivíduos LGBs começaram a fazer uma nova leitura das escrituras, que em geral eram utilizadas como argumentos contra a homossexualidade
(Bowland et al., 2013; Ganzevoort, van der Laan, \& Olsman, 2011; Stamatoulakis \& Nearchou, 2015);

b) Focar em aspectos positivos da religião. consiste em priorizar uma visão de Deus como amoroso, em detrimento de Deus punitivo, e diferenciando sua relação com Deus da relação com a instituição da igreja (Bowland et al., 2013; Ganzevoort et al., 2011; García et al., 2008). Para este fim, estudar teologia a fundo foi importante (Stamatoulakis \& Nearchou, 2015).

c) Compreender a homossexualidade como uma luta interna providenciada por Deus. ideia de que Deus os teria "feito" homossexuais por alguma razão, o que justificaria seus desejos e vivências homossexuais, desculpabilizandoos (Kubicek et al., 2009; Siraj, 2012).

d) Perceber as instituições religiosas como falhas. quando a decisão dos sujeitos das pesquisas foi permanecer nas congregações, ainda que estas apresentassem aspectos de discriminação para as opções sexuais LGBs, sua estratégia foi perceber e se relacionar com a instituição como sendo falha, levada a cabo por pessoas falhas e até mesmo hipócritas. Ao perceberem o ambiente com esse olhar crítico, sentiam-se aliviados da pressão de não serem perfeitos aos olhos dessa comunidade (Bowland et al., 2013; Kubicek et al., 2009; Liboro \& Walsh, 2015).

Outros fiéis LGBs optaram pela estratégia de ativismo religioso, quinta categoria de integração identificada nessa revisão de literatura. $\mathrm{O}$ ativismo refere-se à ação de transformar a igreja de dentro para fora, posicionando-se a favor das causas LGBs e sendo exemplos de liderança nas próprias igrejas, transformando a visão de quem as frequenta acerca da homossexualidade (Bowland et al., 2013).

Muitos homossexuais descritos nos estudos acreditam que a homossexualidade é pecado e que religião e homossexualidade são dois mundos difíceis ou mesmo impossíveis de conciliar. Além disso, creem que a revelação da orientação sexual pode levar ao ostracismo em suas comunidades e famílias de origem. Sendo assim, optam pela sexta estratégia de integração, qual seja, a manutenção de uma das identidades - a identidade religiosa ou a homossexual. As formas encontradas para este fim foram duas: a) optar por abandonar a afiliação religiosa, preservando a identidade homossexual (e.g. Itzhaky \& Kissil, 2015; Jaspal, 2012; Jeffries et al., 2014; Lalich \& McLaren, 2010; Wood \& Conley, 2014); ou b) manter a identidade religiosa em detrimento da orientação sexual ou tentativa de supressão desta (e.g. Kissil \& Itzhaky, 2015; Siraj, 2012; Trammell, 2015; Wood \& Conley, 2014), seja por meio da terapia de conversão sexual (Figueroa \& Tasker, 2013; Ganzevoort et al. 2011; Itzhaky \& Kissil, 2015; Lalich \& McLaren, 2010) ou do casamento heterossexual (Itzhaky \& Kissil, 2015; Lalich \& McLaren, 2010; Kissil \& Itzhaky, 2015). As terapias de mudança de orientação sexual e casamento heterossexual foram sugeridos a partir da busca de aconselhamento com os superiores, na esperança de 
que ambas as estratégias revertessem a homossexualidade. Além disso, como estratégia de supressão da orientação homossexual, foram realizados rituais de limpeza e/ou ações para compensar um possível mau comportamento que fez com que a homossexualidade recaísse sobre eles (Itzhaky \& Kissil, 2015), além do exorcismo (Ganzevoort et al., 2011). Em conjunto, essas estratégias podem apresentar tentativas bastante extremas de integração da R/E. Em um ponto encontra-se o abandono da afiliação religiosa para que se possa viver a homossexualidade livremente; e em outro, tentativas de abafar ou suprimir a orientação homossexual, uma vez que a dimensão religiosa/espiritual tem primazia na vida dessas pessoas.

A sétima e penúltima estratégia de integração da religiosidade/espiritualidade e a orientação sexual é nomeada de negação. Implica, por exemplo, em manter-se estudando e focar na carreira para não ter que lidar com as obrigações religiosas e culturais, como casar e ter filhos (Itzhaky \& Kissil, 2015; Jaspal, 2012; Kissil \& Itzhaky, 2015; Lalich $\&$ McLaren, 2010). Além disso, implica na tendência de atribuir o desejo homossexual a forças externas, ao invés de identifica-los como inerentes a si (Trammel, 2015).

Por fim, a oitava estratégia de integração identificada foi a realização de práticas espirituais individuais, tais como oração, meditação e leitura de material religioso (García et al., 2008; Love, Bock, Jannarone, \& Richardson, 2005). Nesse caso, as práticas espirituais parecem relacionadas eminentemente a uma jornada pessoal, desvinculada de uma vivência coletiva (grupal).

$\mathrm{R} / \mathrm{E}$ como fator de risco e/ou proteção para lésbicas, gays e bissexuais. Essa categoria sumariza e discute os artigos que têm como fio condutor a descrição da religiosidade/espiritualidade como fator de risco e/ou proteção, e de que forma esses fatores se manifestam na vida de LGBs.

$\mathrm{AR} / \mathrm{E}$ pode desempenhar um lugar de referência quanto à segurança, fortalecimento da autoestima e suporte social na vida de indivíduos LGBs quando diminuem o impacto que as adversidades (e.g. homofobia) podem ter sobre suas vidas. No entanto, a R/E também pode se apresentar como fator de risco quando, por exemplo, LGBs fazem parte de alguma religião ou têm uma vivência espiritual que condena sua orientação sexual, gerando uma dissonância cognitiva e causando intensa turbulência emocional (Dahl \& Galliher, 2012) porque potencializa a ocorrência de desfechos negativos ou indesejáveis que comprometem a saúde, bem-estar e relacionamentos sociais do indivíduo (Cowan et al., 1996).

Dessa forma, têm-se os seguintes desfechos: aumento ou redução de ideação/tentativa de suicídio; aumento ou redução no engajamento em comportamento sexual de risco, como a exposição ao HIV/AIDS; aumento do estresse religioso; melhora na aceitação da orientação sexual; aumento do estigma e isolamento social ou aumento do sentimento de pertencimento/suporte social; aumento da depressão/sentimentos de inadequação ou sua diminuição e melhora no manejo de situações difíceis da vida.

Os estudos ressaltam que pertencer a minorias sexuais (Lytle, De Luca, Blosnich, \& Brownson 2014), bem como a homonegatividade internalizada presente no contexto religioso (Gibbs \& Goldbach, 2015) aumenta a chance de ideação suicida. Além disso, a presença do estigma fomentado pela igreja pode provocar baixa autoestima, criando uma atmosfera de silêncio gerada sobre as questões sexuais de homens que fazem sexo com homens (HSHs), deixando-os vulneráveis aos comportamentos sexuais de risco e ao contato com a transmissão do HIV/AIDS (Watkins et al., 2015). Nos dois casos (seja para a ideação suicida ou comportamentos sexuais de risco), o impacto é ainda maior quando advém dos grupos que deveriam oferecer suporte e salvaguardar seus direitos (Dahl \& Galliher, 2012; Mavhandu-Mudzusi \& Sandy, 2015). Por outro lado, também se encontrou estudos que mostram que o engajamento em igrejas inclusivas ou práticas religiosas diminui os índices de ideação/tentativa de suicídio (Kralovec et al., 2012), e reduz o envolvimento sexual com múltiplos parceiros, diminuindo comportamentos sexuais de risco, tal como maior exposição ao HIV/AIDS, e uso de drogas (Foster et al., 2011; Hatzenbuehler, Pachankis, \& Wolff, 2012).

O estresse religioso, por sua vez, acontece quando há dissonâncias cognitivas entre $\mathrm{R} / \mathrm{E}$ e a homossexualidade. Nesse caso, a pressão internalizada das expectativas alheias sobre o caráter pecaminoso da homossexualidade tende a gerar sofrimento nos indivíduos LGBs (Mbetbo, 2013; Siraj, 2012). Por outro lado, a participação nos rituais xamânicos, do Santo Daime e do Ile ale efu l'ase, por meio da ingestão do Ayahuasca1, ajudou os participantes da pesquisa a integrarem melhor sua sexualidade e se dizerem mais confortáveis com sua orientação sexual (Cavnar, 2014).

Tan (2005) identificou que altos índices de bem-estar espiritual- ou seja, o estabelecimento de uma relação positiva com espiritualidade, sentido da vida e transcendência- em homossexuais funcionam como fator de ajustamento, contribuindo para melhora na aceitação da própria orientação sexual, aumento da autoestima e sentimento de pertencer a uma comunidade. Esses desfechos sugerem que um maior autoconhecimento, aceitação das diferenças e conseguir incorporar os valores religiosos podem auxilia-los a serem pessoas mais justas, honestas e compassivas (Dahl \& Galliher, 2012).

O engajamento em igrejas inclusivas também foi considerado fator de proteção para diminuição da depressão e aumento do sentimento de pertencimento e suporte social

1 Aayahuasca é uma infusão vegetal psicoativa da Amazônia. Tipicamente, provoca poderosas visões, assim como alucinações em todas as demais modalidades de percepção. Essas experiências geralmente se associam a insights pessoais, ideações intelectivas, reações afetivas e experiências espirituais e místicas profundas. Também se observam alterações dos parâmetros básicos da experiência - identidade pessoal, conexão com o mundo exterior e temporalidade (Shanon, 2002) 
(Gattis, Woodford, \& Han, 2014); ao passo que crescer ou pertencer a comunidades religiosas que rejeitam a homossexualidade teria o efeito oposto, aumentando os índices de depressão e de sentimentos de inadequação (Dahl \& Galliher, 2012; Mavhandu-Mudzusi \& Sandy, 2015). Conforme se pode perceber, a participação em comunidades religiosas pode representar um fator de risco e/ou de proteção, dependendo da forma como ela encara/concebe a homossexualidade, mas também de como os indivíduos LGBs percebem sua participação nela. Ademais, salienta-se a importância de não se perder de vista o aspecto espiritual presente na religião, reduzindo a ideia de suporte religioso ao pertencimento grupal (Hamblin \& Gross, 2014).

Os resultados dos estudos sugerem que quando os sistemas de fé e orientação sexual colidem, e quando a família e os contextos sociais não são favoráveis, eles podem desafiar a construção de um sentimento positivo de identidade LGB, funcionando como verdadeiros fatores de risco à saúde física e mental desses indivíduos. No entanto, é importante considerar que a perspectiva quanto a esses fatores deve ser processual (Cowan et al., 1996), dependendo, portanto, de variáveis individuais, desenvolvimentais e de contexto, que podem exacerbar o risco ou favorecer a proteção (Sowe, Brown, \& Taylor 2014). Dessa forma, ainda quando os contextos pareçam induzir ao risco, uma vivência religiosa/espiritual positiva pode se mostrar como suporte eficiente e gerador de crescimento.

Homonegatividade internalizada (HI). O termo homonegatividade contempla os aspectos sexistas do preconceito relativo à população LGB, considerando que para além da aversão à orientação sexual, está presente uma percepção engessada e normatizadora dos papéis de gênero. Por homonegatividade internalizada, portanto, entende-se o autodesprezo que lésbicas, gays e bissexuais poderiam sentir por si próprios (Gato, Carneiro, \& Fontaine, 2011). Este foi o termo utilizado nos quatro artigos que compõem essa categoria.

Em um grupo de homens que fazem sexo com homens e que se autodeclaram cristãos, verificou-se que os maiores índices de HI estão relacionados a uma menor frequência de revelação da homossexualidade ("saída do armário"), bem como ao estabelecimento de estados mentais negativos e a comportamentos sexuais de risco, tais como o não uso de preservativos e engajamento sexual com múltiplos parceiros (Wilkerson et al., 2012). Segundo os autores, a exposição ao ensino religioso que condena a homossexualidade, contribui para o aumento da HI e, consequentemente, diminui a frequência de "saídas do armário". De forma semelhante, a exposição à religiosidade - islâmica, cristã e judia - é considerada como a preditora de maior sentimento de vergonha e homofobia internalizada para LGBs; sendo que a magnitude dessa relação é tanto maior quanto mais importante forem os paradigmas religiosos para essas pessoas (Melazde \& Brown, 2015)

Barnes e Meyer (2012) confirmam os achados anteriores, ao relacionar saúde mental com afiliação religiosa e homofobia internalizada. Os resultados de sua pesquisa confirmaram a hipótese geral de que nas religiões nas quais a orientação sexual LGB não é bem-vinda verificase um maior grau de homofobia internalizada entre seus adeptos. No entanto, ao contrário do que hipotetizaram, curiosamente não houve correlação significativa entre religiões não inclusivas e mal-estar psicológico. Além disso, os autores apontaram grande adesão dos pesquisados LGBs a permanecerem nas instituições religiosas em que estão inseridos desde a infância, ainda que se trate de instituições não inclusivas e até com postura discriminatória acerca dos homossexuais. Esta escolha se dá diante do conforto espiritual e apoio comunitário sentido pelos sujeitos e que lhes são difíceis de descartar (Barnes \& Meyer, 2012).

Acerca da relação entre $\mathrm{R} / \mathrm{E}$, comportamentos sexuais de risco e HI de homens (negros americanos) que fazem sexo com homens (HSHs), verificou-se que religiosidade e espiritualidade se associaram de forma diferente à HI. Enquanto os sujeitos com alta religiosidade apresentavam índice mais elevado de HI, aqueles com alta espiritualidade demonstravam maior aceitação de sua orientação sexual, corroborando a importância de que as dimensões religiosidade e espiritualidade devam ser vistos como construtos diferentes (Smallwood et al. 2015).

Os estudos analisados nesta categoria avançam sobre a compreensão da presença e dos efeitos da HI na vida de gays, lésbicas e bissexuais que vivenciam a R/E. Verificou-se que viver em contextos que internalizam desde cedo que o ideal social é heteronormativo pode gerar nos homossexuais um autoconceito negativo e influenciar sua vida religiosa/ espiritual. No entanto, os efeitos da homofobia internalizada para a vivência da religiosidade e da espiritualidade parecem ser diferentes, influenciando mais negativamente a primeira do que a segunda dimensão.

Prática clínica. A R/E configura-se como uma dimensão importante da constituição psíquica dos sujeitos e com frequência chega à clínica psicológica. Ainda que ambos, paciente e terapeuta, compactuem das mesmas crenças religiosas/espirituais, deve-se considerar que as formas de vivenciar a religião/espiritualidade se dão de formas muito particulares, de acordo com cada indivíduo que as experimenta (Henning-Geronasso \& Moré, 2015).

Nos artigos que integram essa categoria, são descritos aspectos-chave da prática clínica na visão de LGBs que vivenciam e/ou têm demandas acerca de sua religiosidade/ espiritualidade relacionadas à orientação sexual. Importante sublinhar que todos os artigos nesta categoria são de origem norte americana, contemplando, portanto, experiências desse contexto, no qual terapias de conversão (ou reorientação) sexual são permitidas. No Brasil, a partir da Resolução 
001/1999, os psicólogos foram proibidos de colaborar com eventos e serviços que proponham tratamento e cura das homossexualidades (Conselho Federal de Psicologia [CFP], 1999).

Um primeiro estudo investigou especificamente as formas em que o aconselhamento/terapia contribuíram para os esforços de desenvolvimento de identidade de clientes LGBs relacionados com $\mathrm{R} / \mathrm{E}$. Os participantes do estudo relataram experiências de aconselhamento positivas e negativas a partir de como foi conduzida a questão da identidade sexual e identidade religiosa/espiritual pelo conselheiro (Goodrich et al., 2015).

Com relação às experiências negativas, os participantes relataram perceber que alguns terapeutas não tinham muito treinamento nem conhecimento da importância das identidades sexual ou religiosa/espiritual em sessão; onde se comunicavam a partir de vieses pessoais sobre questões sexuais ou religiosas/espirituais; e, ainda, causaram danos com os esforços de uma terapia de conversão sexual. Em contrapartida, muitos participantes relataram terapeutas que foram acolhedores, autênticos e abertos. Muitos se identificaram com os terapeutas em termos de identidades sexuais, religiosas/espirituais ou políticas, e tiraram benefícios dessa identificação (Goodrich et al., 2015).

Ainda sobre o relacionamento terapêutico, o estudo de Bowland et al. (2013) enfocou a perspectiva de gays e lésbicas cristãos - que consideram que fizeram bem a integração entre religiosidade e orientação sexual- acerca das habilidades desejadas para o terapeuta que trabalhe com demandas religiosas/ espirituais e de orientação sexual. Dentre estas, citam-se: ter consciência de si, lidar com suas próprias questões e lutas internas; ter perspectiva histórica, considerar que a depender da geração em que cresceu e saiu do armário, as respostas sociais são variáveis; ter atitudes de não julgamento; e não limitar a pessoa à sua orientação sexual.

Foram identificados artigos específicos sobre terapia de reorientação sexual, isto é, processos clínicos que anunciam um tratamento de mudança de orientação sexual, de homossexual para heterossexual. Um dos estudos examinou preditores (reações familiares, meio social e fundamentalismo religioso) da participação dos sujeitos nesse tipo de tratamento. Os resultados encontraram que as reações familiares, fundamentalismo religioso e auto identificação como pessoas espiritualizadas, seriam preditoras do engajamento em terapias de conversão sexual (Maccio, 2010). O autor encontrou que aqueles com valores religiosos conservadores, podem ter dificuldade em conciliar suas crenças e sua orientação sexual homossexual. Tal dissonância pode resultar em sua tentativa de alterar sua sexualidade. A associação da espiritualidade como preditor para a realização de terapia de conversão sexual foi um achado inesperado, explicada pelas hipóteses de que os sujeitos que se consideraram espiritualizados podem ainda manter os valores fundamentalistas de uma religião que outrora participaram, ou mesmo que atenderam a terapias de conversão sexual à época do engajamento em uma religião organizada (Maccio, 2010). O artigo pontua ainda que assistir a esse tipo de terapia pode causar danos aos sujeitos na medida em que a necessidade para mudar a orientação sexual ou identidade tem a ver com obter a aceitação de outros, colocando de lado os próprios processos de autoaceitação e autoestima.

Em outro estudo sobre a terapia de conversão sexual foram analisados os esforços de mudança de orientação sexual por 1.612 indivíduos que são membros atuais ou antigos da Igreja de Jesus Cristo dos Santos dos Últimos Dias - mórmons (Bradshaw et al., 2015). Os fatores associados às tentativas de mudança de orientação sexual incluíram níveis mais elevados de contato com a religião ortodoxa desde a infância e baixo índice de aceitação pelas famílias, além de comunidades pouco inclusivas. Os participantes, ao demandarem como objetivo na terapia a mudança da orientação sexual, relataram baixa eficácia para quase todos os métodos. No entanto, alguns resultados benéficos foram encontrados quando- durante o processo terapêutico com esse objetivo de conversão sexual- houve a aceitação do sentimento de atração pelo mesmo sexo e, consequentemente, redução da depressão e ansiedade. Os resultados fundamentam a conclusão de que a orientação sexual é altamente resistente às tentativas explícitas para a mudança e que esses tratamentos são relatados em grande número como ineficazes ou prejudiciais pelos participantes.

Um aspecto em comum dos artigos é a necessidade de um manejo respeitoso no sentido de evitar imposições a partir de imperativos pessoais, culturais e religiosos seja por parte do terapeuta, dos padres e pastores a quem esses sujeitos buscam. Alguns autores discutem que a maior dificuldade que o profissional enfrenta ao entrar em contato com a religiosidade (e orientação sexual) de seu cliente é a de lidar com seus próprios preconceitos.

\section{CONSIDERAÇÕES FINAIS}

Este estudo teve como objetivo apresentar um panorama da produção científica, nacional e internacional, acerca da experiência religiosa/espiritual de LGBs, privilegiando-se os estudos que focalizavam a perspectiva do indivíduo LGB acerca da $\mathrm{R} / \mathrm{E}$.
Verificou-se o predomínio de estudos acerca das estratégias de integração R/E e homossexualidade, seguidos pelos estudos com foco na religiosidade/espiritualidade como fator de risco e/ou proteção para LGBs. Assim sendo, constatou-se que - quando há uma dissonância cognitiva 
entre o paradigma da religião e a orientação sexual -, LGBs religiosos/espirituais se utilizam de estratégias para que sua orientação sexual e vida religiosa/espiritual convivam harmoniosamente, ou elegem vivenciar um aspecto em detrimento do outro. Nesse processo, as características individuais e a forma como as comunidades religiosas encaram a homossexualidade, norteiam se a religiosidade/ espiritualidade funcionará como entidade protetiva e/ ou de risco na vida dessas pessoas. Ademais, a literatura parece ser consensual no que toca ao efeito negativo da homofobia sobre a vivência religiosa dos LGBs, uma vez que age limitando-a, embora não impeça a vivência da espiritualidade, considerada como de foro mais íntimo e subjetivo (menos social/comunitária) que a religiosidade. Outro consenso refere-se à ineficácia e prejuízos trazidos pelas chamadas terapias de conversão sexual à vida dos LGBs.

Entende-se, a partir da leitura dos textos e da demanda clínica, que investigar a experiência religiosa/espiritual de LGBs a partir da sua própria percepção é uma tarefa importante, visto a centralidade dessa dimensão na vida dos seres humanos, levando em consideração os números expressivos de brasileiros que se declaram filiados a alguma religião (IBGE, 2010). Ao mesmo tempo, trata-se de uma tarefa que implica lidar com muitos desafios, seja aqueles advindos da comunidade acadêmica (pouco afeita ao estudo de temáticas relacionadas à religiosidade/espiritualidade), da clínica psicológica que apresenta dificuldades frente à esta demanda (Cambuy, Amatuzzi, \& Antunes, 2006) ou ainda do preconceito da sociedade e das comunidades religiosas, que ainda consideram a homossexualidade um pecado e/ou passível de reversibilidade, o que significaria dizer que o que é passível de ser revertido e, portanto, não necessitaria de políticas públicas ou de proteção, e sim da administração de um tratamento adequado.

A forma como os princípios religiosos influenciam o cotidiano de vida brasileiro, ultrapassa a esfera de igrejas, comunidades ou congregações, e adentra o congresso nacional, através de uma frente parlamentar que milita na contramão da garantia de direitos à população LGBT. Lembrando que estas manifestações ganham espaço na política devido à própria característica conservadora do congresso e da sociedade que o elege e não apenas do poder desta específica frente parlamentar (Trevisan, 2013). Enquanto for este ainda o contexto, pesquisas como a presente, se fazem necessárias.

Diante de uma literatura inteiramente internacional, este artigo busca contribuir com a literatura nacional acerca da descrição e compreensão da experiência religiosa/espiritual de LGBs. Espera-se que os conteúdos aqui tratados possam ser úteis para as pesquisas e intervenções voltadas ao público LGB, visto que nossa literatura não contempla, por exemplo, os caminhos das estratégias de integração R/E, e pouco ainda, se deteve em investigar os mecanismos a partir dos quais a $\mathrm{R} / \mathrm{E}$ pode funcionar como um fator de risco ou de proteção para LGBs. Ao mesmo tempo, a literatura analisada fornece evidências importantes que podem embasar/fortalecer o debate acerca dos aspectos deletérios da homofobia e das terapias de conversão sexual.

Finalmente, em termos de estudos futuros, sugere-se a realização de pesquisas empíricas que investiguem as estratégias utilizadas por lésbicas, gays, bissexuais e os/ as transgêneros, com base nas categorias de análise aqui sistematizadas e em outras que venham porventura surgir.

\section{REFERÊNCIAS}

Bardin, L. (1995). Análise de Conteúdo (L. Reto \& A. Pinheiro, Trad.). São Paulo: Edições 70/ Livraria Martins Fontes (Originalmente publicado em 1977)

Bastos, C. N. R. S (2014) "O segredo de uma família feliz". Representações sobre família entre as testemunhas de Jeová em Santo Estêvão/BA (1970-2001) (Dissertação de mestrado). Universidade Federal de Feira de Santana, BA, Brasil.

Bowland, S. E, Foster, K, \& Vosler, N. R (2013) Culturally competent and spiritually sensitive therapy with lesbian and gay Christians. Social Work, 58(4), 321-332. doi: 10.1093/ sw/swt037.

Bruscagin, C. (2004). Família e religião. In C. M. O. Cerveny (Org.), Família, comunicação, divórcio, mudança, resiliência, deficiência, lei, bioética, doença, religião e drogadição (pp. 163-186). São Paulo: Casa do Psicólogo.

Busin, V. M. (2011). Religião, sexualidades e gênero. Revista de Estudos da Religião, 11(1), 105.

Cambuy, K., Amatuzzi, M. M., \& Antunes, T. A. (2006). Psicologia clínica e experiência religiosa. Revista de Estudos da Religião, 3, 77-93.

Carvalho, A. D. Z. D. (2014). Entre Política e Religião: Diferenciação funcional e laicidade seletiva no Brasil. Rivista Krypton-RomaTrE-Press. Recuperado de https://papers.ssrn. $\mathrm{com} /$ sol3/papers.cfm?abstract_id $=2476886$
Conselho Federal de Psicologia. (1999). Resolução CFP N 001/99 de 22 de Março de 1999 "Estabelece normas de atuação para os psicólogos em relação à questão da Orientação Sexual". Recuperado de http://site.cfp.org.br/wp-content/ uploads/1999/03/resolucao1999_1.pdf

Cohen, K. M., \& Savin-Williams (2014). "Saindo do armário" para si mesmo e para os outros. In J. Drescher, M. E. Barber, \& P. Levounis (Orgs.), O livro de casos clínicos GLBT (pp. 36-51). Porto Alegre: Artmed.

Cowan, P. A., Cowan, C. P., \& Schulz, M. S. (1996). Thinking about risk and resilience in families. In E. M. Hetherington \& E. A. Blechman (Eds.), Stress, coping and resiliency in children and families (pp. 1-38). New Jersey: Erlbaum.

Domínguez, D. G., Bobele, M., Coppock, J., \& Peña, E. (2015). LGBTQ relationally based positive psychology: An inclusive and systemic framework. Psychological Services, 12(2), 177185. doi: $10.1037 / \mathrm{a} 0038824$

Ferreira, R. J., \& da Silva, M. G. (2015). A organização eclesiástica da Comunidade Cristã Nova Esperança: Entre acolhimentos e desacolhimentos. HORIZONTE, 13(40), 2292-2307.

Gato, J., Carneiro, N. S., \& Fontaine, A. M. (2011). Contributo para uma revisitação histórica e crítica do preconceito contra as pessoas não heterossexuais. Revista Crítica e Sociedade, 1(1), 139-167. 
Heerman, M., Wiggins, M., \& Rutter, P. (2007). Creating space for spiritual practice: Pastoral possibilities with sexual minorities. Pastoral Psychology, 55, 711-721. doi:10.1007/s11089-0070085-y.

Henning-Geronasso, M. C., \& Moré, C. L. O. O. (2015). Influência da religiosidade/espiritualidade no contexto psicoterapêutico. Psicologia: Ciência e Profissão, 35(3), 711-125. doi: 10.1590/1982-3703000942014.

Instituto Brasileiro de Geografia e Estatística. (2010). Características gerais da população, religião e pessoas com deficiência. Recuperado de http://biblioteca.ibge.gov.br/visualizacao/ periodicos/94/cd_2010_religiao_deficiencia.pdf

Itzhaky, H., \& Kissil, K. (2015). "It's a horrible sin. If they find out, I will not be able to stay": Orthodox jewish gay men's experience living in secrecy. Journal of Homosexuality, 62, 621-643. DOI: 10.1080/00918369.2014. 988532

Jesus, F. W. A. (2010). A cruz e o arco-íris: Refletindo sobre gênero e sexualidade a partir de uma "igreja inclusiva" no brasil. Ciencias Sociales y Religión/Ciências Sociais e Religião, 12(12), 131-146.

Louceiro, L. M. (2007). As variedades da experiência religiosa de William James revisitadas. Cognitio-Estudos Revista Eletrônica de Filosofia, 4(2), 103-120.

Machado, M. D. D. C., Barros, M. L. D., \& Piccolo, F. D. (2010). Judaísmo e homossexualidade no Rio de Janeiro: Notas de uma pesquisa. Religião \& Sociedade, 30(1), 11-31.

Natividade, M. (2006). Homossexualidade, gênero e cura em perspectivas pastorais evangélicas. Revista Brasileira de Ciências Sociais, 21(61), pp. 115-223.

Natividade, M. (2010). Uma homossexualidade santificada? Etnografia de uma comunidade inclusiva pentecostal. Religião e Sociedade, 30(2), 90-121.
Nunes, M. C. (2013). Família cristã e homoafetiva na modernidade religiosa. Em III Seminário Internacional Enlaçando Sexualidades. Universidade do Estado da Bahia.

Oliveira, E. N. (2015). Adoção por casais homoafetivos em uma visão crítico-jurídica e social. Revista Lexmax, 3(3), 101-107.

Oswald, R. F. (2002). Resilience within the family networks of lesbians and gay men: Intentionality and redefinition. Journal of Marriage and Family, 64(2), 374-383.

Pinto, E. B (2009), Espiritualidade e religiosidade: Articulações. Revista de Estudos da Religião, dezembro, 68-83.

Rodrigues, L. P. M. (2009). "Uma abominação à vista do Senhor": O discurso fundamentalista da sexualidade Mórmon (Trabalho de Conclusão de Curso). Universidade Federal da Paraíba, João Pessoa, PB, Brasil.

Scorsolini-Comin, F. (2014). Guia de orientação para a iniciação científica. São Paulo, SP: Atlas.

Shanon, B. (2002). The antipodes of the mind: Charting the phenomenology of the ayahuasca experience. Cambridge, MA: Oxford University Press.

Souza, R. M. D. (2013). A salvação da homossexualidade: Reflexões sobre uma comunidade inclusiva cristã (Monografia). Universidade de Brasília, Brasília, DF, Brasil.

Trevisan, J (2013), A Frente Parlamentar Evangélica: Força política no estado laico brasileiro. Numen: Revista de Estudos e Pesquisa da Religião, 16(1), 29-57.

Vilaça, H., \& Oliveira, M. J. (2015). Clivagens e cumplicidades entre e a Igreja Católica e o estado: O casamento entre pessoas do mesmo sexo. Sociologia, Problemas e Práticas, (78), 29-47.

Walsh, F. (2005). Fortalecendo a resiliencia familiar. São Paulo: Roca.

\section{LITERATURA REVISADA}

Barnes, D. M., \& Meyer, I. H (2012) Religious affiliation, internalized homophobia, and mental health in lesbians, gay men, and bisexuals, American Journal of Orthopsychiatry, 82(4), 505-515. doi: 10.1111/j.1939-0025.2012.01185.x

Bradshaw, K., Heaton, T. B., Decoo, E., Dehlin, J. P., Galliher, R. V., \& Crowell, K. A. (2015). Religious experiences of GBTQ Mormon males. Journal for the scientific study of religion, 54(2), 311-329. doi: 10.1111/jssr.12181

Cavnar, C. (2014). The effects of ayahuasca ritual participation on gay and lesbian identity. Journal of Psychoactive Drugs, 46(3), 252-260. doi: 10.1080/02791072.2014.920117.

Dahl, A. L., \& Galliher, R. V. (2012). LGBTQ adolescents and young adults raised within a Christian religious context: Positive and negative outcomes. Journal of Adolescence, 35, 1611-1618. doi:10.1016/j.adolescence.2012.07.003.

Dehlin, J. P., Galliher, R. V., Bradshaw, W. S., Hyde, D. C., \& Crowell, K. A. (2015). Sexual orientation change efforts among current or former LDS church members. Journal of Counseling Psychology, 62(2), 95-105. doi: 10.1037/cou0000011.

Figueroa, V., \& Tasker, F. (2013). "I always have the idea of $\sin$ in my mind. ...". Family of origin, religion, and Chilean young gay men. Journal of GLBT Family Studies, 10(3), 269-297. doi: 10.1080/1550428X.2013.834424.

Foster, M. L., Arnold, E., Rebchook, G., \& Kegeles, S. M. (2011). 'It's my inner strength': spirituality, religion and HIV in the lives of young African American men who have sex with men. Culture, Health \& Sexuality: An International Journal for Research, Intervention and Care, 13(9), 1103-1117. doi: 10.1080/13691058.2011.600460.
Ganzevoort, R. R., van der Laan, M., \& Olsman, E. (2011). Growing up gay and religious. Conflict, dialogue, and religious identity strategies. Mental Health, Religion \& Culture, 14(3), 209-222. doi: 10.1080/13674670903452132.

García, D., Gray-Stanley, J., \& Ramires-Valles, J. (2008). "The priest obviously doesn't know that i'm gay": The religious and spiritual journeys of Latino gay men. Journal of Homosexuality, 55(3), 411-436. doi: 10.1080/00918360802345149.

Gattis, M. N., Woodford, M. R., \& Han, Y. (2014). Discrimination and depressive symptoms among sexual minority youth: Is gay-affirming religious affiliation a protective factor? Archives Sexual Behavior, 43, 1589-1599. doi: 10.1007/s10508-0140342-y.

Gibbs, J. J., \& Goldbach, J. (2015). Religious conflict, sexual identity, and suicidal behaviors among LGBT young adults. Archives of Suicide Research, 19(4), 472-488. doi: 10.1080/13811118.2015.1004476.

Goodrich, K. M., Buser, J. K., Luke, M., \& Buser, T. J. (2015). Spiritual and sexual identity: Exploring lesbian, gay, and bisexual clients' perspectives of counseling. Journal of Homosexuality, 63(6), 783-807. doi: 10.1080/00918369.2015.1112192.

Hamblin, R. J., \& Gross, A. M. (2014). Religious faith, homosexuality, and psychological well-being: A theoretical and empirical review. Journal of Gay and Lesbian Mental Health, 18(1), 67-82. doi: 10.1080/19359705.2013.804898.

Hatzenbuehler, M. L., Pachankis, J. E., \& Wolff, J. (2012). Religious climate and health risk behavior in sexual minority youths: A population-based study. American Journal of Public Health, 102(4), 657-663. doi: 10.2105/AJPH.2011.300517. 
Jaspal, R. (2012). 'I never faced up to being gay': Sexual, religious and ethnic identities among British Indian and British Pakistani gay men. Culture, Health and Sexuality: An Internacional Journal for research, Intervention and Care, 14(7), 767- 780. doi: 10.1080/13691058.2012.693626.

Jeffries, I. V. W. L., Okeke, J. O., Gelaude, D. J., Torrone, E. A., Gasiorowicz, M., Oster, A. M., ... Bertolli, J. (2014). An exploration of religion and spirituality among young, HIV-infected gay and bisexual men in the USA. Culture Health \& Sexuality: An International Journal for Research, Intervention and Care, 16(9), 1070-1083. doi: 10.1080/13691058.2014.928370.

Kissil, K., \& Itzhaky, H. (2015). Experiences of the marital relationship among orthodox Jewish gay men in mixedorientation marriages. Journal of LGBT Family Studies, 11(2), 151-172. doi: 10.1080/1550428X.2014.900659.

Kralovec, K., Fartacek, C., Fartacek, R., \& Ploderl, M. (2012). Religion and suicide risk in lesbian, gay and bisexual Austrians. Journal of Religion and Health, 53(2), 413-423. doi: 10.1007/ s10943-012-9645-2.

Kubicek, K., McDavitt, B., Carpineto, J., Weiss, G., Iverson, E., \& Kipke, M. D. (2009). "God made me gay for a reason": Young men who have sex with men's resiliency in resolving internalized homophobia from religious sources. Journal of Adolescent Research, 24(5), 601-633. doi: 10.1177/0743558409341078.

Lalich, J., \& McLaren, K. (2010). Inside and outcast: Multifaceted stigma and redemption in the lives of gay and lesbian Jeovah's Witnesses. Journal of Homosexuality, 57, 1303- 1333. doi: 10.1080/00918369.2010.517076.

Love, P., Bock, M., Jannarone, A., \& Richardson, P. (2005). Identity interaction: Exploring the spiritual experiences of lesbian and gay college students. Journal of College Student Development, 46(2), 193-209. doi: 10.1353/csd.2005.0019.

Liboro, R. M., \& Walsh, R. T. G. (2015) Understanding the irony: Canadian gay men living with HIV/AIDS, their catholic devotion, and greater well-being. Journal of Religious Health, 55(2), 650-670. doi: 10.1007/s10943-015-0087-5.

Lytle, M. C., De Luca, S. M., Blosnich, J. R., \& Brownson, C. (2014). Associations of racial/ethnic Identities and religious affiliation with suicidal ideation among lesbian, gay, bisexual, and questioning individuals. Journal of Affective Disorders 1(178), 39-45. doi: 10.1016/j.jad.2014.07.039.

Maccio, E. M. (2010). Influence of family, religion, and social conformity on client participation in sexual reorientation therapy. Journal of Homosexuality, 57(3), 441-458. doi: 10.1080/00918360903543196

Mavhandu-Mudzusi, A. H., \& Sandy, P. T. (2015). Religion-related stigma and discrimination experienced by lesbian, gay, bisexual and transgender students at a South African rural-based university. Culture, Health \& Sexuality, 17(8), 1049-1056. doi: 10.1080/13691058.2015.1015614.
Mbetbo, J. M. (2013). Internalised conflicts in the practice of religion among kwandengue living with HIV in Douala, Cameroun. Culture, Health \& Sexuality: An International Journal for Research, Intervention and Care, 15(sup1), 76-87. doi: 10.1080/13691058.2013.779025.

Melazde, P., \& Brown, J. (2015). Religion, sexuality, and internalized homonegativity: Confronting cognitive dissonance in the Abrahamic religions. Journal of Religious Health, 54(5), 1950-1962. doi: 10.1007/s10943-015-0018-5.

Shilo, G., Yossef, I., \& Savaya, R. (2015) Religious coping strategies and mental health among religious jewish gay and bisexual men. Archives of Sexual Behavior, 45(6), 1551-1561. doi: 10.1007/s10508-015-0567-4

Siraj, A. (2012) "I don't want to taint the name of Islam": The influence of religion on the lives of Muslim lesbians. Journal of Lesbian Studies, 16(4), 449- 467. doi: 10.1080/10894160.2012.681268.

Smallwood, S. W., Spencer, M., Ingram, L. A., Thrasher, J. F., \& Thompson-Robinson, M. V. (2015). Examining the relationships between religiosity, spirituality, internalized homonegativity, and condom use among African American men who have sex with men in the deep south. American Journal of Men's Health, 11(2), 196-207, doi: 10.1177/1557988315590835.

Sowe, B. J., Brown, J., \& Taylor, A. J. (2014). Sex and the sinner: Comparing religious and nonreligious same-sex attracted adults on internalized homonegativity and distress. American Journal of Orthopsychiatric, 84(5), 530-544. doi: 10.1037/ ort0000021.

Stamatoulakis, K. K., \& Nearchou, F. (2015). Homosexuality and priesthood: Conflict in the life of a Norwegian woman. Procedia-Social and Behavior Sciences, 185, 160-164. doi: 10.1016/j.sbspro.2015.03.406

Tan, P. (2005). The importance of spirituality among gay and lesbian individuals. Journal of Homosexuality, 49(2), 135-144. doi: 10. 1300/j082v49n02 08.

Trammel, J. Y. (2015). "Homosexuality is bad for me": An analysis of homosexual Christian testimonies in Christianity today magazine. Journal of Media and Religion, 14, 1-15. doi: 10.1080/15348423.2014.971560.

Watkins Jr, T. L., Simpson, C., Cofield, S. S., Davies, S., Kohler, C., \& Usdan, S. (2015). The relationship between HIV risk, high-risk behavior, religiosity, and spirituality among black men who have sex with men (MSM): An exploratory study. Journal of Religion and Health, 55(2), 535-548. doi: 10.1007/ s10943-015-0142-2.

Wilkerson, J. M., Smolenski, D. J., Brady, S. S., \& Rosser, B. R. S. (2012). Religiosity, internalized homonegativity and outness in Christian men who have sex with men. Sexual and Relationship Therapy, 27(2), 122-132. doi: 10.1080/14681994.2012.698259.

Wood, A. W., \& Conley, A. H. (2014). Loss of religious or spiritual identities among the LGBT population. Counseling and Values, 59, 95-111. doi: 10.1002/j.2162-oo7X.2014.00044X. 